\title{
How do they solve it? An insight into the learner's approach to the mechanism of physics problem solving
}

\author{
Balasubrahmanya Hegde \\ Department of Physics, BASE, Bangalore, India \\ B. N. Meera* \\ Department of Physics, Bangalore University, Bangalore, India \\ (Received 12 August 2011; published 9 March 2012)
}

\begin{abstract}
A perceived difficulty is associated with physics problem solving from a learner's viewpoint, arising out of a multitude of reasons. In this paper, we have examined the microstructure of students' thought processes during physics problem solving by combining the analysis of responses to multiple-choice questions and semistructured student interviews. Design of appropriate scaffoldings serves as pointers to the identification of student problem solving difficulties. An analysis of the results suggests the necessity of identification of the skill sets required for developing better problem solving abilities.
\end{abstract}

DOI: 10.1103/PhysRevSTPER.8.010109

PACS numbers: 01.40.Fk, 01.40.Ha, 01.40.gb

\section{INTRODUCTION}

One of the important objectives while teaching physics is to ensure that students effectively learn the content and efficiently use their knowledge base to analyze new and novel physics situations. One of the means which can ensure the aforesaid objective is to make students acquire good problem solving abilities. Testing problem solving abilities of a student serves as both a diagnostic tool (that tests the content learned) and an evaluative tool (that tests their ability to use what is learned). Many researchers have examined various aspects of problem solving, the earliest works dealing with the explicit definition and construction of a problem [1,2]. With the emergence of interest in physics problem solving by physics education researchers, considerations and approaches to problem solving specific to physics were necessitated [2-4]. A well-structured physics problem leads to a unique and unambiguous solution as against the solution to a problem in general connotation.

Problem solving in general and physics problem solving, in particular, encapsulate several intriguing aspects. The identification and exploration of these aspects can offer a multipronged tool to address the issues pertaining to difficulties faced by many students for problem solving. Having identified that, it is important to realize that these aspects possess an element of subjectivity and often are not even tangible in a given context. Nevertheless, research has indicated that taking cognizance of the following aspects

\footnotetext{
*Author to whom correspondence should be addressed. bnmeera@gmail.com

Published by the American Physical Society under the terms of the Creative Commons Attribution 3.0 License. Further distribution of this work must maintain attribution to the author(s) and the published article's title, journal citation, and DOI.
}

of problem solving may yield significant and practical improvements in problem solving abilities of students.

\section{GOAL OF PROBLEM SOLVING}

Learning goals associated with physics problem solving are multifaceted. The primary objective being attainment of effective understanding of physics, the subsidiary ones are those that train a student to develop an ability to connect various ideas and concepts in a self-consistent way, to apply their understanding in various situations encountered, and/or even simply to train the learner in problem solving. The process of concept learning leading to the concept application with the goal of culminating in expertise in problem solving is a productive and appending cycle. Hence, problem solving plays a very important role in building cognitive cognizance for it has all the potential to strengthen the other two processes associated with the learning.

\section{NATURE OF PROBLEM}

The above said goal, attainment, however, depends on the nature of the problem. Even though there is an element of subjectivity, a broad based categorization of physics problems in principle is possible and their respective learning outcomes can be identified [5]. Unambiguous labeling of the nature of a problem is often not feasible because a given physics problem may be composed of one or more of the characterizing elements. Students' problem solving inclination gets influenced by the nature of the problem and has a differing consequence, both in instruction and learning. For example, a problem which predominantly requires the usage of an equation may pose difficulty to the student merely because of failure to recall the relevant equation. The need of the equation being the primary requirement, any other input to the student may not turn 
out to be of much use. Such a feature is recorded to be one of the major issues plaguing problem solving abilities of students. The Rolodex-equation hunting method not only makes the student skip the concept features of a problem, but also promotes rote learning [6-9]. Yet another issue is the influence on problem solving ability that gets affected by the nature of different representational formats. Different representational formats are found to have varied appeals to learners. The usage of different representational formats during instruction and the intrinsic style of a student are also contributing factors guiding student approach and ability in problem solving [10-13].

A physics problem that has a strong component of mathematical manipulation is often known to compound the difficulty in problem solving. A lack of ability to coordinate a variety of skills including mathematical processing, computation, blending ancillary information with math, and reading out physical implications from math and vice versa [14] is naturally an impediment to becoming a good problem solver. Also, an intrinsic or acquired fear of mathematics presents a formidable impediment in problem solving. In spite of mathematical interplay being a significant component in problem solving, its role is seldom recognized by novice learners. The transition of a student from a concept learner to a problem solver requires a strong appreciative approach to the interplay of mathematics in physics learning. The influence and consequence of learning mathematics "only as mathematics" but not as a tool in physics has been investigated in various contexts $[14,15]$.

The idea that knowledge structure of a learner is a critical factor influencing the ability to solve a physics problem necessitated the detailed examination of processes pertaining to problem solving. Many researchers have correlated students' understanding of physics to the knowledge structure students are associated with $[16,17]$. It appears that a good conceptual clarity can enable a student to solve a problem successfully. However, research results show that problem solving ability need not emerge as a consequence of conceptual clarity alone [17-19]. Therefore, an explicit training in physics problem solving may help the student to acquire the skills needed. Also, establishment of a procedural framework for problem solving in physics requires a deeper understanding of the necessary skills [20-24]. Research suggests that successful physics problem solvers identify the deeper features of physics relevant to the problem rather than use surface features to solve the problem, thus presenting the distinction between approaches of "experts and novices" [25-28]. Heller et al. record that a coherent group dynamics leads to a better ability in physics problem solving $[23,24]$. The role played by different types of problems in the light of considerations for instructional and evaluation strategies have been examined [29-33]. The works of Van Huvelen report better problem solving abilities after the usage of an instructional tool known as overview, case study [34]. The primary aim of all of the above approaches is to understand processes adopted by students in physics problem solving so as to generate instructional and pedagogical perspectives. More recent works in this research area have attempted to obtain a detailed understanding of problem solving processes. A deeper insight into problem solving processes of a learner sheds light not only on their learning difficulties but also on how students process information pertaining to physics. As observed by researchers, the individual knowledge structure analysis is more significant because it brings forth the issues of self-regulation and self-correction modes of a learner [18,35-40]. Research has also shown that one of the parameters tampering students' learning curve is the lack of dynamic feedback mechanism from textbooks, a consequence of which is that attempting real life physics problems is always a matter of difficulty to students as against the end of chapter, non-context-rich, problems [29]. The cognitive issues and the epistemological beliefs of students that influence the learning ecosystem have been studied by many physics education researchers and experts from other relevant fields [41-45].

This brings forth the issue of explicit inclusion of an expertlike approach to problem solving during instruction. Such an approach calls for the identification of skill sets which are to be honed for problem solving $[1,46]$. As mentioned earlier, research has shown a marked difference in the approach of novice learners and expert learners during problem solving. An attempt to teach an expertlike problem solving approach by teaching explicit skill of categorization based on deeper conceptual features rather than surface features to a novice learner has shown that the skill sets necessary for effective problem solving are many. This skill set in combination with rigorous explicit training in problem solving, involving problems of all aforesaid varieties, can promote problem solving abilities of students [47].

Experience and research findings suggest a dominant indisposition towards physics problem solving. However, what is not disclosed completely is the information about the nature and cause of students' difficulties in physics problem solving. In the absence of these details any effort to help students develop problem solving skills may remain incomplete and ineffective. Earlier attempts to probe students difficulties associated with problem solving are based on the analysis of student responses to questionsmostly in multiple-choice question (MCQ) format. The method, however, is an "averaged" method for it does not take the strengths and weaknesses of an individual into consideration, but approaches the issue in a general and statistical way. The recent trend in physics education research is to use the analysis of data drawn from such a statistical approach and then choose a few problems for student clinical interview and analyze the microstructure of student responses. These responses reveal not just the 
content deficiency of students but also the inconsistencies in their knowledge structure and the associated cognitive issues. These internal inconsistencies prompted us to investigate in detail the problem solving mechanism by using a combination of analysis of the MCQ responses and analysis of elucidated reactions to structured interviews. We are not interested in evaluating students' ability to solve physics problems. We require that students go through the problem solving activity exposing inconsistencies in their knowledge structure.

\section{METHODOLOGY}

Acknowledging the fact that the type of the problem is crucial in deciphering the nature of difficulty involved in problem solving, in this research we have selected a problem which validates the following components: the role of equation hunting given a problem and the role of mathematical manipulation when multiple variables are involved in a problem. Our earlier work on a problem which can be termed as a bridge problem (a problem that requires the use of concepts from different domains of physics) has shown the results which support the common observation that a large number of students exhibit compartmentalized learning [44]. Their knowledge base appears to be fragmented, which can possibly be the cause of a major block in the majority of the students' problem solving ability. The inability of students to draw conclusions based on everyday observations appears to be an issue predominantly arising out of inconsistencies in their beliefs about physics in the classroom and the physics of the world around us. The lack of mathematical manipulation skills in an altered and unfamiliar context is also evident [44].

The present study is composed of two phases. The first phase involves an analysis of responses of a large number of students to selected physics problems. In the second phase, this was then followed by an in-depth personal interview of a smaller number of students. A set of carefully contrived questions, which were in the MCQ format, was designed for the first phase of the research. The objective of designing the pilot test in the MCQ format was its established advantage in testing the breadth of knowledge within a group of students. A set of 22 questions was designed with each question having a clear objective. Each of these questions has some of the components discussed above. This phase was expected to shed light on student learning difficulties, more specifically in the domain of physics problem solving. The students' responses obtained during this first phase were analyzed. Based on these responses, relevant pointers pertaining to physics problem solving have been identified. No statistical analysis of the data of the first phase was carried out - the responses are intended to serve as inputs to the second phase.

The responses to the MCQ test are indicative of the nature of the student difficulty; however, a microstructure of the understanding of problem solving processes is more important for our research, which is the intended objective to be served in the second phase. This is realized by a detailed and well structured student interview. The analysis of student responses to the MCQ test feeds the design of the interview protocol. We are interested in the nature of processes the student resorts to during physics problem solving rather than determining the success or failure to solve a physics problem. Hence we have provided appropriate scaffoldings (serving as rubrics [48]) for the student during their interview. These scaffoldings not only allow a student to continue to progress towards the solution of the problem but also help us to understand the nature of perceived difficulties. The considerations that formulate this critical step of student interview protocol design will be discussed in detail in the context of the problem chosen for this study.

In the first phase of our research, the sample group was composed of nearly 300 students in the age group of 19-21 years who are students of a three year undergraduate course in science. The undergraduate science course involves learning chemistry and mathematics in addition to physics, with equal emphasis on all three subjects. The students who participated in the study were from different colleges, yielding a heterogeneous sample which was expected to unfold various aspects of problem solving skills of students learning physics.

In order to understand the microstructure of the underlying cognitive process of physics problem solving, the interview mode, which is standard and accepted practice in physics education research, has been adopted. For the second phase of personal interviews, among the problems used in the first phase, we selected five problems with representative problem characterizing elements as discussed earlier. In this paper, we discuss the results of one of these problems. The protocol for the interviews was carefully designed to examine the influence of those characterizing elements. This is achieved by posing questions or making observations during the conduct of the interview that serves as rubrics.

Such an approach, based on student interview, requires formulation of carefully constructed hints of increasing strength, which may even depend on student responses. These ladder-type clues are known as scaffoldings. Being a custom-made support for the construction of new skills, a support that can be easily disassembled when no longer needed, or a structure that allows for the accomplishment of some goal that would otherwise be either unattainable or quite cumbersome to complete [49], it is natural that more of such detailed scaffoldings are needed for a novice problem solver. Even though the primary goal of scaffolding is to help the learners to channelize their thinking, the same scaffolding can also serve as a tool to understand learning disabilities since the nature of the scaffolding the student uses and the method in which they use it shall 
reflect on their thinking process. Though the usefulness of scaffolding is largely dependent on student competency, its effectiveness in building and strengthening the thought process necessary for problem solving is well supported. There are no quantitative methods to estimate the usefulness or level of scaffoldings; the design is largely based on the inputs from the first stage of our research.

Another important requirement of the question design is to ascertain that a question has the intended effect. A tool to accomplish this process is the validation interview which identifies whether a question conveys the intended meaning of the interviewer and whether the given scaffolding has the desired impact. Based on the careful analysis of the validation interview, the questions and the scaffoldings were restructured wherever necessary. The scaffoldings were provided at appropriate stages to ensure the progress of thought processes towards the solving of the problem. In the data interview phase, ten students were interviewed for the question chosen for this study. The average time for an interview was about $40 \mathrm{~min}$., though we did not put a stringent constraint on the time duration for any interview. The interviews, conducted in a specially designed laboratory, were recorded using a video camera and an electronic writing pad. The recordings have been transcribed and analyzed.

\section{A. First phase}

The question presented in the first phase is given below. The energy radiated by a black body $(E)$ is related to its absolute temperature $(T)$ through the equation, $E=A \sigma T^{4} t$. A spherical black body radiates at a power $P$. When its radius is halved and temperature is doubled, the power is
(A) halved
(B) unaltered
(C) doubled
(D) quadrupled

Research results indicate that students resort to hunting for the relevant equation as the important first step while solving a problem. Not being able to recall the equation could be one of the major reasons for not solving the problem. It is important to eliminate this possibility and obtain deeper insight into the processes in problem solving in physics. Therefore, the relevant equation has been embedded in the statement of the problem.

TABLE I. MCQ analysis.

\begin{tabular}{lc}
\hline \hline Options & Responses \\
\hline (A) halved & $7 \%$ \\
(B) unaltered & $25 \%$ \\
(C) doubled & $33 \%$ \\
(D) quadrupled & $28 \%$ \\
\hline \hline
\end{tabular}

290 students took the test of which 270 students attempted the question with 20 not attempting it. The response of the students is presented in Table I.

The nature of the distribution of the responses does not establish any clear dominant aspect of difficulty in solving this problem. Only $28 \%$ of the students answered correctly and the others answered wrongly. Obviously, providing the relevant equation alone appears not to help in problem solving. The analysis of the MCQ responses presents us with the following tasks. The first one is the necessity to validate the observation that "equation hunting" is not the only perceived difficult part of physics problem solving. The second is the requirement to probe more into the way students attempt interlinking different physical concepts (power and energy in the present problem context). In addition to these, the role and mode of mathematical processing when multiple variables are involved also needs to be probed.

The scaffoldings provided during the interview determine the usefulness of interview outcome. Research findings show that students rarely have a tendency to look for the underlying physical principles while solving a problem. Therefore, presenting the relevant physical principle to the problem solver is a weak scaffolding. The stronger tendency for a student is to hunt for the relevant equation. Any help in this direction is, therefore, a strong scaffolding. In the design of the interview protocol, the weakest scaffolding was provided first. The categorization of a scaffolding as a weak scaffolding or a strong one is not really absolute. The sequencing order of the scaffoldings was generated taking into account the analysis of MCQ responses and also validation interview results.

\section{B. Second phase}

The details of the different stages of the interview protocol design are discussed below.

Step 1-The question was presented as given below.

A spherical black body radiates at a power $P$. When its radius is halved and temperature is doubled, its power is
(A) halved
(B) unaltered
(C) doubled
(D) quadrupled

The question "did not" contain the relevant equation. Though equation hunting is perceived as the primary step to solve a problem, it is important to investigate the actual stage at which a student needs to recall the equation.

Step 2.- Students who did not attempt to solve were suggested to use Stefan's law. The probability of a problem solving mechanism being activated is high once a triggering point is provided. This triggering point can be the act of reminding one of the association of the problem situation to a familiar real world situation or even mentioning the name of the relevant physical principle in the form of a 
familiar law or simply providing the relevant equation. Research shows that novice learners tend to attempt a problem based on its surface features rather than on the deeper relevant physical principles. Therefore, we have used the scaffolding in the form of the name of the physical principle to be used as the trigger-weakest scaffolding first.

Step 3.-For those who did not benefit successfully from the above scaffolding, the necessary physical principle was provided in the form of verbal statement of Stefan's law. The question was rephrased as follows.

According to Stefan's law, energy radiated per unit time by a black body is directly proportional to fourth power of its absolute temperature and its surface area. A spherical black body radiates at a given power. When its radius is halved and temperature is doubled, its power is
(A) halved
(B) unaltered
(C) doubled
(D) quadrupled

In this form of the question, Stefan's law has been stated in terms of energy. However, formal instructions present Stefan's law in terms of power (which is rate of change of energy). The objective of not stating Stefan's law in terms of power is to investigate student's ability to interlink related concepts in physics-energy to power and vice versa.

Step 4.- Students who were not successful in using the scaffolding mentioned above were presented with the question which stated Stefan's law in verbal form in terms of power. Students were then asked to represent the verbal form of Stefan's law provided to them in the mathematical form. Research has shown that lack of multiformat representational skill is an important issue which limits the ability of a student to comprehend a given idea when represented in various representational formats. Verbal representation, mathematical representation, graphical representation, etc. do not appeal equally to all learners, whereas they serve to provide enhancement to the clarity of ideas for experts. This important cognitive issue is expected to be one of the important factors that limits a novice learner from translating given data into procedural variables to be used along with an equation or formula.

Step 5.-In the next step, students who could not use the above scaffolding successfully were presented with a mathematical equation representing Stefan's law (in terms of energy). They were then asked to identify the symbols in the mathematical form of Stefan's law. In physics, use of symbols is necessitated by the inevitable use of mathematics for quantifying physical ideas. An equation in physics is a relation between different physical quantities-not a mere relation between symbols. More importantly, it is inevitable that there exists degeneracy in the use of these symbols. It is often observed that students associate symbols to incorrect physical quantities and read the relevant physics wrongly. It is thus interesting to probe the strength of association of symbols and physical quantities.

Step 6.-Finally, the students are given Stefan's law in the mathematical form in terms of power and energy. This step is equivalent to providing them the relation between power and energy. With all the available data and access to the relevant "equation," students only needed to use their arithmetic skills to arrive at the correct answer. A strong manipulation skill in mathematics and an ability to handle simultaneous variation of multiple variables is of course a desired integral aspect of learning physics and the lack of it can be a major deterrent in problem solving.

The structure of the interview protocol is summarized in the mind map shown in Fig. 1. This summary map is a very useful tool for strategy management during the interview.

Mind map.-There ought to be instances when the student answers a question correctly and indeed there were some. The answering process often is associated with some feedback coming from the transcription of the notes the student makes (on the electronic note pad) during the interview process. But these need further probing since this by itself is not often very instructive as they are incomplete and incoherently fragmented. As discussed, each of the above scaffoldings comprises an objective with a core idea. When the student answers correctly, we

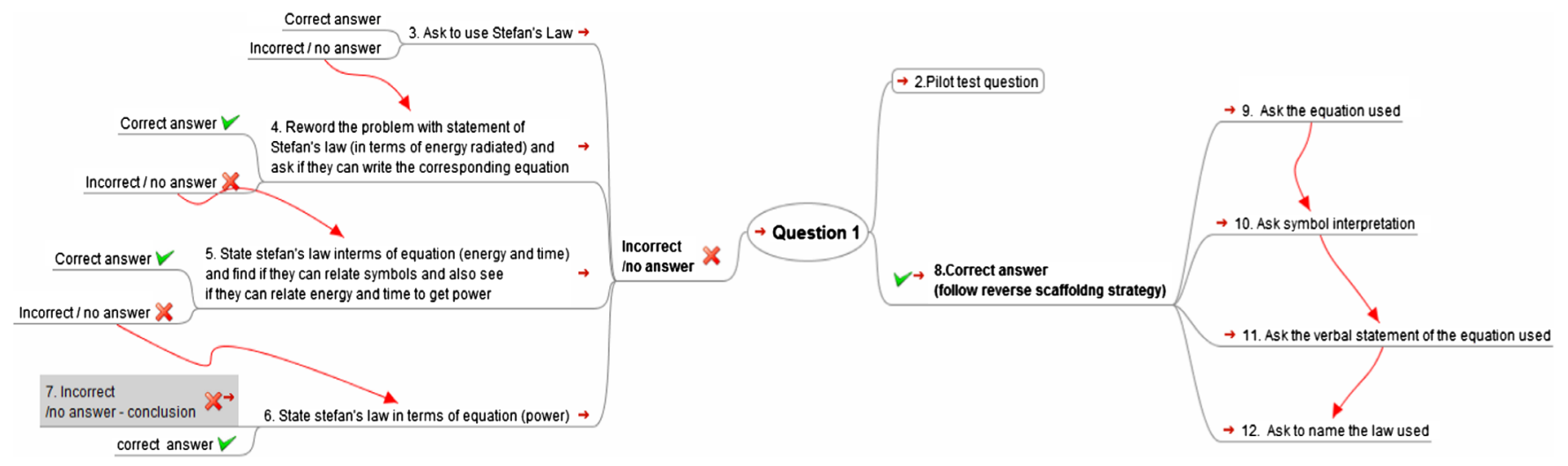

FIG. 1 (color online). Mind map for scaffolding strategy management. 
first attempt to see the equation used by the student as this is the core idea for the strongest scaffolding. The interview, when students answer successfully, proceeds exactly in the reverse order-the questions are in terms of the idea behind the strongest scaffolding (the relevant equation) first and in terms of the idea behind the weakest scaffolding (the relevant physical principle) last.

\section{RESULTS AND DISCUSSIONS}

Step 1.-When presented, only two students solved it successfully without any scaffolding adding support to the results of the first phase that showed 28\% correct responses. It has been observed that those who solved the problem successfully without any scaffolding looked for an equation as a first step towards the solution and key words like energy, black body, radiation, and temperature provided them the trigger to search for the relevant equation to be used. Interestingly, students' recognition of the physical principle was only through the equation they identified. For them, perhaps, an equation is a stand-alone entity — not the translation of a physical principle.

Interviewer: "Stefan's law was in your mind?"

Student: "First it was not. First I wrote the expression for the power ... they say energy, black body. Radius and temperature are given. Thus Stefan's law has to be used."

The students who solved successfully found little difficulty in handling simultaneous variation of multiple variables, apparently because of their exposure to similar problems. The epistemological idea that understanding involves knowing when to use an equation and how to use an equation plays an important role in the progress to the solution to a physics problem.

Student: “. .. now $E=\sigma A e T^{4} t \ldots A$ is $\pi r^{2} \ldots$ so $\sigma \pi r^{2} e T^{4} t$. There are two constants. Now $t$ is also constant ... because ... per second ... or $P$ is proportional to $r^{2} T^{4}$ ".

Indeed equation hunting appears to be the dominant strategy adopted by students while solving physics problems. However, a detailed analysis of results of MCQ response to the situation which had the equation embedded in the problem did not show an increased ease of problem solving. Hence the role of availability of an equation in problem solving needs further validation. Even yet another student who identified the physical principle as Stefan's law, however, did not progress due to a predominant difficulty in handling situations dealing with proportionality. Such a situation could either arise due to problems in language comprehension or difficulty in mathematical reasoning.

One student identified that the power depends on temperature, before asking for any scaffolding.

"... when the temperature increases, the power increases ...."

However, the student could not capitalize this correct idea to solve the problem. The student could identify neither the name of the physical principle nor the principle governing the solution.
Step 2.-The scaffolding which suggests a student use Stefan's' law-without stating the law explicitlytriggered only one student to proceed, but unsuccessfully. He tried to relate power to absolute temperature and surface area. He related them as follows:

“... this is actually based on Stefan's law. ...power is directly proportional to fourth root of temperature."

However, when probed, he could not connect the power radiated to the surface area. The remaining seven students expressed their inability in recalling the statement of Stefan's law. This result necessitates a critical examination of the supposition that problem solving can be facilitated by providing an effective triggering mechanism in terms of relevant physical principle (or equally well, the name of the physical law).

Step 3.-The inability of some students in taking the problems towards solution with the above scaffolding prompted us to provide the statement of Stefan's law. The statement of Stefan's law was provided in verbal form (not as an equation) - in terms of energy and time.

The ability to interpret the voice of physics (involving language and concepts) in terms of language of physics (mathematics) is an important step towards the understanding of physics and it also accelerates problem solving abilities in physics. Hence, translating the statement of the law into a desired mathematical form should function as an effective scaffolding. We have two intentions associated with this step. The first is to examine their ability in translating a verbal form of a physical principle into a mathematical equation. The second is to examine the effectiveness of such a translation towards the problem solution. The progress of a student at this juncture depends on how well they translate the statement of the principle as an equation.

One student used this scaffolding successfully, with a little assistance in distinguishing change in radius from change in area. However, stating Stefan's law in verbal form did not appear to help six students as they could not successfully write the mathematical form of Stefan's law.

The existing instructional strategies rarely pay obeisance to the development of skills that establish the navigational abilities from conceptual platform to mathematical framework. Our research data reveal this as a possible hindrance to problem solving abilities.

Step 4.-For those who could not use the verbal form of Stefan's law, the next scaffolding was provided. In this step, we presented the mathematical form of Stefan's law in terms of energy and time. This step has three objectives. First it is intended to function as strong scaffolding for the problem solution. The second is to examine the nature of students' association of symbols to physical quantities used in the context of a given equation. The third is to examine students' concept networking in terms of interrelated physical quantities-in this context the relation between energy and power. 
The question presented was in terms of power whereas the scaffolding presented was in terms of energy and time. In the course of the formal instruction, Stefan' law is stated in terms of the power radiated by a blackbody. The idea of power as rate of energy transfer is learned qualitatively as well as quantitatively-more so in the context of mechanics.

This scaffolding helped one student to solve it successfully. Another student used this step to proceed but unsuccessfully. The students' confusion, however, resulted as a result of recalling the formula for surface area of a sphere erroneously.

A: " $\ldots$ the surface area is $\pi r^{2} \ldots$ "

B: " $\ldots$ the surface area is $(4 / 3) \pi r^{3} \ldots$ "

However, he corrected himself when his attention was drawn to the fact that area involves "length square." The remaining five students could not use this scaffolding successfully.

This data presented us a very important surprising result. Our hypothesis that equation hunting is the most critical step in problem solving seems to be only partially justified. However, we cannot ignore another important consideration which might have constrained them, which, in this case, is the possible inability to incorporate ideas learned in different contexts. In the formal instruction mode, Stefan's law is stated in terms of power. Although students are introduced to the concept of power as "work done per unit time" in the context of Newtonian mechanics, it is the fragmented and compartmentalized learning mode which limits their ability to extend the knowledge to an altered situation. The necessary ability to relate power and energy, even though trivial, cannot be underestimated. Surprisingly, “... energy is power by time ..." was one of the frequent answers when students were required to relate energy and time.

Another important revelation is the existence of confusion in relating different symbols used in the context of an equation to the relevant physical quantities. The degeneracy arising out of the representation of different physical quantities with the same symbol is inevitable. For example, symbols like $\sigma, e, n, T$, etc. find different meanings depending on the context. This "identity crisis" is an inevitable part of physics learning. For a novice learner, this degeneracy can be disadvantageous, for the probability of not relating a symbol to the appropriate physical quantity in a given context is high. As a consequence, the association of the related physical quantity to the appropriate physical process is not decoded. This needs an important consideration for it has all the potential to suppress the triggering of a problem solving mechanism. For an expert learner, however, it is clear that these symbols are just artificial constructs relating different representational formats.

Step 5.-In the final step, we presented the scaffolding which provided the equation for Stefan's law in terms of power. This step ensured that the student has the complete equation. With this scaffolding, this problem is as good as any end of the chapter plug and chug problem. However, the difficulty arising out of the necessity of mathematical manipulation, in this context the idea of proportional reasoning with simultaneous variation of multiple variables, may prevent a student from completing the solution in spite of having all the necessary information.

None of the students who were presented with this scaffolding were able to solve the problem completely. Three students could not differentiate the radius becoming half from surface area becoming one-fourth. An explicit help had to be rendered.

A detailed analysis of the above results provides us with a glimpse of the attributes necessary to develop and enhance problem solving ability. The ever-haunting question of whether the students' approach to problem solving is through search of equation or concept resurfaces here again. The analysis of the interview chronicles indicates the absence of the necessary strategies needed for problem solving. Once these are identified, the necessary skill sets, finite in number, that are context and content independent can be identified and explicit attention for the development of those can be ensured by a balanced methodology which guarantees a "process over content" approach.

\section{CONCLUSIONS}

Our research suggests a methodology for examining the parameters which act as deterrents while solving a physics problem. The existing and practiced instructional strategies often concentrate on building a conceptual framework along with the relevant mathematical rigor. However, often a student fails to construct their ideas beyond definitions and statements of laws which get exemplified in the context of problem solving. Our research probes in detail the microstructure of student thought processes in the course of problem solving. Our semistructured interviews along with appropriately designed scaffoldings bring out the exact nature of difficulties which otherwise do not get exposed. These lead us to the following possible conclusions.

As a first step in problem solving, it is logical to identify the physical principle applicable to the situation. However, the weak association of students' conceptual framework to the physical principles acts as a major deterrent in problem solving. Most often, the physical terms in the problem statement act as trigger for the search of an equation and the inability to do so may hinder the problem solving completely. Also, the inability to proceed even when a student has access to the relevant equations is an intriguing observation. The weakness students' possess in connecting the symbols to the physical quantities can be attributed to a tendency to look at an equation in physics not as a relation between physical quantities but merely as a mathematical equation. Another important parameter 
which can play a significant role in limiting problem solving abilities is the lack of mathematical manipulation skills.

A factor whose influence cannot be undermined in studies of this kind is the role of domain vulnerability of the problem solver in the problem context. A student who is already unclear of concepts in a domain of physics may not get much help from scaffoldings. The scaffolding in such cases may not instigate the student towards solution. Another limitation, here, is the nonuniqueness of scaffoldings, which cannot be sequenced. The absence of such a hierarchical structure may render a set of scaffoldings (generated for a question, designed for a learner) unusable in some other context. This being a limitation of the probing methodology, learner-independent but systemdependent unique scaffoldings can hardly be designed. However, the absence of such a hierarchical structure does not deter a student from continuing with the solution.

The most important issue of "how to improve problem solving abilities" necessarily needs explicit attention. A possible technique is to develop and teach strategies and protocols. However, these strategies are nonunique since they are strongly context dependent. Our research and other research results support the argument that exposure and practice in problem solving is a necessary requirement for a better problem solving ability. However, research has also established that solving a large number of problems alone does not necessarily develop a conceptual clarity and may not really help in solving a novel problem. Hence, practice without an emphasis on the subtleties of the problem solving may not lead to better problem solving abilities. Identification of skill sets therefore becomes a necessary step for better problem solving abilities. Good problem solving can happen if a deliberate attempt is made to build the necessary skill sets. However, it is evident that these cannot be taught as stand-alone skill sets, as they are often contextual and hence can only be an embedded attribute in training. A highly focused practice in problem solving along with care for the development of the necessary skills will therefore be a necessary and sufficient condition to promote better problem solving abilities.
[1] G. Polya, How to Solve It (Princeton University Press, Princeton, NJ, 1945).

[2] A. Newell and H. A. Simon, Human Problem Solving (Prentice Hall, Englewood Cliffs, NJ, 1972).

[3] J.H. Larkin and F. Reif, Understanding and teaching problem-solving in physics, Int. J. Sci. Educ. 1, 191 (1979).

[4] F. Reif, J. H. Larkin, and B. C. Bracket, Teaching general learning and problem solving skills, Am. J. Phys. 44, 212 (1976).

[5] E. Yerushalmi and E. Cohen, K. Heller, P. Heller, and C. Henderson, Instructors' reasons for choosing problem features in a calculus-based introductory physics course, Phys. Rev. ST Phys. Educ. Res. 6, 020108 (2010).

[6] T. J. Bing and E.F. Redish, Analyzing problem solving using math in physics: Epistemological framing via warrants, Phys. Rev. ST Phys. Educ. Res. 5, 020108 (2009).

[7] Lillian C. McDermott and Edward F. Redish, Resource Letter: PER-1: Physics Education Research, Am. J. Phys. 67, 755 (1999).

[8] Dong-Hai Nguyen and N. Sanjay Rebello, Students' difficulties with integration in electricity, Phys. Rev. ST Phys. Educ. Res. 7, 010113 (2011).

[9] P. B. Kohl and N.D. Finkelstein, Patterns of multiple representation use by experts and novices during physics problem solving, Phys. Rev. ST Phys. Educ. Res. 4, 010111 (2008).

[10] Louis Deslauriers and Carl Wieman, Learning and retention of quantum concepts with different teaching methods, Phys. Rev. ST Phys. Educ. Res. 7, 010101 (2011).

[11] P. B. Kohl and N. D. Finkelstein, Student representational competence and self-assessment when solving physics problems, Phys. Rev. ST Phys. Educ. Res. 1, 010104 (2005).

[12] Patrick B. Kohl and Noah D. Finkelstein, Effects of representation on students solving physics problems: A fine-grained characterization, Phys. Rev. ST Phys. Educ. Res. 2, 010106 (2006).

[13] P. B. Kohl and N. D. Finkelstein, Patterns of multiple representation use by experts and novices during physics problem solving, Phys. Rev. ST Phys. Educ. Res. 4, 010111 (2008).

[14] Dong-Hai Nguyen and N. Sanjay Rebello, Students' difficulties with integration in electricity, Phys. Rev. ST Phys. Educ. Res. 7, 010113 (2011).

[15] Dong-Hai Nguyen and N. Sanjay Rebello, Students' understanding and application of the area under the curve concept in physics problems, Phys. Rev. ST Phys. Educ. Res. 7, 010112 (2011).

[16] F. Reif and J.I. Heller, Knowledge structure and problem solving in physics, Educ. Psychol. 17, 102 (1982).

[17] I. A. Halloun and D. Hestenes, The initial knowledge state of college physics students, Am. J. Phys. 53, 1043 (1985).

[18] D. Hammer, Students' beliefs about conceptual knowledge in introductory physics, Int. J. Sci. Educ. 16, 385 (1994).

[19] E. F. Redish, J.M. Saul, and R. N. Steinberg, Student expectations in introductory physics, Am. J. Phys. 66, 212 (1998).

[20] David Melony, in Handbook of Research in Science Teaching and Learning, edited by D. Gabel (MacMillan Reference Books, New York, 1994). 
[21] R. K. Boyle and D. P. Maloney, Effect of written text on usage of Newton's third law, J. Res. Sci. Teach. 28, 123 (1991).

[22] P. S. Shaffer and L. C. McDermott, Research as a guide for curriculum development: An example from introductory electricity. Part II: Design of an instructional strategy, Am. J. Phys. 60, 1003 (1992).

[23] P. Heller, R. Keith, and S. Anderson, Teaching problem solving through cooperative grouping. Part 1. Group versus individual problem solving, Am. J. Phys. 60, 627 (1992).

[24] P. Heller and M. Hollabaugh, Teaching problem solving through cooperative grouping. Part 2. Designing problems and structuring groups, Am. J. Phys. 60, 637 (1992).

[25] M. T. H. Chi, P. S. Feltovich, and R. Glaser, Categorization and representation of physics problems by experts and novices, Cogn. Sci. 5, 121 (1981).

[26] R. Elio and P. B. Scharf, Modeling novice-to-expert shifts in problem-solving strategy and knowledge organization, Cogn. Sci. 14, 579 (1990).

[27] J. H. Larkin, J. McDermott, D. Simon, and H. A. Simon, Expert and novice performance in solving physics problems, Science 208, 1335 (1980).

[28] P. B. Kohl and N.D. Finkelstein, Patterns of multiple representation use by experts and novices during physics problem solving, Phys. Rev. ST Phys. Educ. Res. 4, 010111 (2008).

[29] R. Bhaskar and H. A. Simon, Problem solving in semantically rich domains: An example from engineering thermodynamics, Cogn. Sci. 1, 193 (1977).

[30] D. E. Trowbridge and L.C. McDermott, Investigation of student understanding of the concept of velocity in one dimension, Am. J. Phys. 48, 1020 (1980).

[31] D. Palmer, The effect of context on students' reasoning about forces, Int. J. Sci. Educ. 19, 681 (1997).

[32] F. M. Goldberg and J.H. Anderson, Student difficulties with graphical representations of negative values of velocity, Phys. Teach. 27, 254 (1989).

[33] B. J. Duch, Problem-based learning in physics: Making connections with the real world, AIP Conf. Proc. 399, 557 (1997).

[34] A. Van Heuvelen, Overview, Case Study Physics, Am. J. Phys. 59, 898 (1991).

[35] C. A. Ogilvie, Changes in students' problem-solving strategies in a course that includes context-rich, multifaceted problems, Phys. Rev. ST Phys. Educ. Res. 5, 020102 (2009).
[36] D. Rosengrant, A. Van Heuvelen, and E. Etkina, Do students use and understand free-body diagrams?, Phys. Rev. ST Phys. Educ. Res. 5, 010108 (2009).

[37] C. Lindstrøm and M.D. Sharma, Link maps and map meetings: Scaffolding student learning, Phys. Rev. ST Phys. Educ. Res. 5, 010102 (2009).

[38] C. Singh, Assessing student expertise in introductory physics with isomorphic problems. I. Performance on no intuitive problem pair from introductory physics, Phys. Rev. ST Phys. Educ. Res. 4, 010104 (2008).

[39] Chandralekha Singh, Assessing student expertise in introductory physics with isomorphic problems. II. Effect of some potential factors on problem solving and transfer, Phys. Rev. ST Phys. Educ. Res. 4, 010105 (2008).

[40] Aaron R. Warren, Impact of teaching students to use evaluation strategies, Phys. Rev. ST Phys. Educ. Res. 6, 020103 (2010).

[41] F. Reif and J. H. Larkin, Cognition in scientific and everyday domains: Comparison and learning implications, J. Res. Sci. Teach. 28, 733 (1991).

[42] David Hammer, Epistemological beliefs in introductory physics, Cogn. Instr. 12, 151 (1994).

[43] D. Hammer et al., Transfer of Learning: From a Modern Multidisciplinary Perspective, edited by J.P. Mestre (Information Age Publishing, Greenwich, CT, 2005), pp. 89-120.

[44] Balasubrahmanya Hegde and B. N. Meera, Technology and Mathematics Education, edited by Sugra Chunawala and Meena Kharatmal (MacMillan, Mumbai, India, 2011), pp. 132-136.

[45] Daniel Dolmert et al., An exploration of university physics students' epistemological mind sets towards the understanding of physics equations, NorDiNa 1, 15 (2007).

[46] Edward de Bono, Lateral Thinking: Creativity Step by Step (Harper and Row, New York, 1970).

[47] Eugenia Etkina, Anna Karelina, and Maria RuibalVillasenor, How long does it take? A study of student acquisition of scientific abilities, Phys. Rev. ST Phys. Educ. Res. 4, 020108 (2008).

[48] S. Murthy, Peer-Assessment of Homework Using Rubrics, in Proceedings of the Physics Education Research Conference, edited by L. Hsu, C. Henderson, and L. McCullough (AIP, Melville, NY, 2007), p. 156-159.

[49] C. Lindstrøm and M. D. Sharma, Teaching physics novices at university: A case for stronger scaffolding, Phys. Rev. ST Phys. Educ. Res. 7, 010109 (2011) 